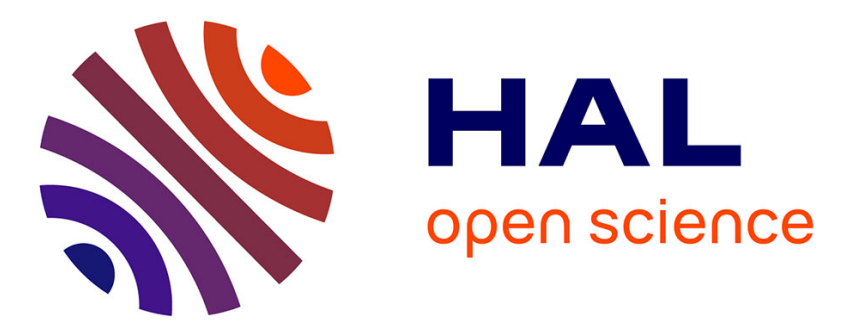

\title{
Notre façon moderne de voir est conditionnée par la camera obscura - Réflexion sur les fondements logiques de la perception et de la mesure
}

Philippe Matherat

\section{- To cite this version:}

Philippe Matherat. Notre façon moderne de voir est conditionnée par la camera obscura - Réflexion sur les fondements logiques de la perception et de la mesure. Intellectica - La revue de l'Association pour la Recherche sur les sciences de la Cognition (ARCo), 2007, 2007/1 (45), pp.167-191. halshs-00243158

\section{HAL Id: halshs-00243158 \\ https://shs.hal.science/halshs-00243158}

Submitted on 7 Feb 2008

HAL is a multi-disciplinary open access archive for the deposit and dissemination of scientific research documents, whether they are published or not. The documents may come from teaching and research institutions in France or abroad, or from public or private research centers.
L'archive ouverte pluridisciplinaire HAL, est destinée au dépôt et à la diffusion de documents scientifiques de niveau recherche, publiés ou non, émanant des établissements d'enseignement et de recherche français ou étrangers, des laboratoires publics ou privés. 


\title{
Notre façon moderne de voir est conditionnée par la camera obscura
}

\author{
Réflexion sur les fondements logiques de la perception et de la mesure
}

\author{
Philippe MATHERAT ${ }^{1}$
}

\begin{abstract}
Résumé. La perspective est-elle un mode de représentation de l'espace parmi d'autres possibles ou bien nous dit-elle une vérité sur l'espace réel ? La photographie nous donne-t-elle un accès direct au réel, ou bien estelle empreinte de la subjectivité du photographe, voire d'une trahison due à l'appareil ? En quoi les mondes virtuels de la synthèse d'image diffèrent du monde réel ? Ces questions nous obligent à nous réinterroger sur la perception visuelle, sur l'origine de nos connaissances à propos de l'espace extérieur, sur la mesure, et sur les liens entre les fondements de la physique et le langage. La vision nous semble être à la fois une comparaison à des représentations et une intention créatrice qui demande une action motrice. Les représentations ne sont pas forcément situées, ni forcément immatérielles. Elles sont prises dans une boucle action/sensation dans laquelle intervient le langage et la culture. Nous ne sommes jamais dispensés d'avoir à imaginer la réalité, même si notre imagination doit être confrontée au réel.
\end{abstract}

Mots-clés : réel-virtuel, représentation, sensation, perception, imagination, logique, physique, mesure, simulation, vision, perspective, photographie.

\begin{abstract}
Our modern way of seeing is governed by the camera obscura - Reflection on the logical foundations of perception and measurement. Is perspective a way of representing space among other possible ways of representation or does it say a truth about real space? Does photography give us a direct access to the real world, or is it influenced by the subjectivity of the photographer, or by a betrayal of the camera? In which respects the virtual worlds of image synthesis are different from real world? These questions drive us to re-examine the visual perception, the origin of our knowledge about the external world, the operation of measurement, the links between foundation of physics and language. To see is to compare representations to sensations and also to act on the external world in a creative way. Representations are not necessarily located in space, and not necessarily constituted of matter. They are in a loop combining action and sensation in which there are also language and culture. We have to imagine reality, even if our imagination has to be tested with the real.
\end{abstract}

Keywords : real-virtual, representation, sensation, perception, imagination, logic, physics, measurement, simulation, sight, perspective, photography.

\section{INTRODUCTION}

Ce texte est structuré en deux parties de formes et de longueurs très différentes :

1. La première partie (la plus courte) a la forme d'un discours scientifique, c'est un cours d'optique photographique, résumé à sa trame. Elle montre le rôle joué par la géométrie de la propagation de la lumière, tant dans la représentation en perspective que pour déduire les paramètres de fonctionnement d'un appareil photographique.

2. La seconde partie a la forme d'une critique de la première partie. Nous serons amenés à explorer les questions de la perception, de l'origine de nos connaissances sur l'espace, de la nature de la mesure en physique. Ceci nous conduira à tenter une réponse à la question : Qu'est-ce que voir ?

L'articulation entre les deux parties est faite par la proposition la lumière se propage en ligne droite. Dans la première partie, cette proposition est utilisée comme une connaissance de physique permettant de décrire le fonctionnement de la chambre photographique. Dans la deuxième partie, nous nous interrogerons sur le statut logique de cette proposition, sur son origine, sur ce qu'elle nous dit, si elle est fondée. Cette interrogation nous

' CNRS - LTCI (UMR 5141) / TELECOM-ParisTech, département Comelec, 46 rue Barrault, 75013 Paris. matherat@telecom-paristech.fr 
conduit inévitablement vers des questions de métaphysique, et vers le rôle du langage. Nous trouverons une issue en proposant de considérer la mesure comme une construction active.

Notons que, dans la deuxième partie, nous ne chercherons pas à assimiler l'oeil à la camera obscura, au contraire. Notre interrogation porte sur l'origine de nos connaissances sur l'espace. La camera obscura intervient par le lien avec la géométrie de la perspective. Elle intervient en outre en suggérant une opposition entre monde extérieur et monde intérieur. Cette opposition, nous en questionnerons le bien-fondé.

\section{LA TRAME D'UN COURS D'OPTIQUE PHOTOGRAPHIQUE}

Nous allons essayer de montrer ici que l'essentiel d'un cours de physique sur le fonctionnement d'un appareil photographique peut être dérivé de façon très simple (se résumant à quelques constructions de géométrie élémentaire) de la seule proposition la lumière se propage en ligne droite. Notre but est donc de dire quelque chose sur la structure du discours du physicien. Nous n'en montrerons que la trame, et nous renverrons le lecteur à des ouvrages spécialisés pour le détail des démonstrations mathématiques, comme par exemple (Gaudart et Albet, 1997).

Nous noterons qu'il n'est nul besoin de détailler la conception des assemblages de lentilles de verre de l'objectif photographique pour traîter presqu'entièrement ce qu'on appelle optique photographique. La quasitotalité de cette optique peut être traîtée en ne considérant que des appareils sans lentille (un simple trou appelé sténopé), puis en ne parlant à propos des lentilles que de leur effet d'association de points conjugués.

Ainsi énoncée, l'optique photographique semblera ne concerner que la boîte, la camera obscura, qui apparaît comme la partie la plus importante de l'appareil photographique.

\section{1. Formation de l'image, perspective}

Répétons l'expérience classique de la camera obscura : construisons une boîte en carton dont un fond est remplacé par une feuille de papier calque et dont le fond opposé est percé d'un petit trou (d'un diamètre d'un demi-millimètre pour une boîte de 20 centimètres de profondeur). L'image qui se forme sur le calque est faible, visible seulement quand on dirige le trou vers un objet très lumineux comme une fenêtre ou le fillament d'une lampe à incandescence (figure 1). Pour mieux voir cette image, il est préférable de recouvrir la tête de l'observateur et la partie arrière de la boîte d'un même voile opaque.

Comment rendre compte de ce que nous observons, comment expliquer la formation de cette image ? Nous dirons à la suite des physiciens que la lumière se propage en ligne droite. Une telle ligne droite est nommée rayon lumineux. Les rayons lumineux remplissent tout l'espace, dans toutes les directions. Habituellement, on ne dessine que ceux qui participent directement à la création de l'image. Mais l'image n'existe que par son contraste, c'est-à-dire le rapport d'intensité lumineuse entre les parties claires et les parties sombres. Ce contraste est limité par l'intensité de rayons dits parasites, nommés ainsi par leur action d'affaiblissement du contraste : ce sont les rayons qui viennent éclaircir les zones sombres de l'image. Il faut donc éliminer ces rayons, c'est la fonction de la paroi de la chambre. On peut ainsi considérer (figure 1) que la chambre noire est un appareil à trier les rayons lumineux.

Faisons un classement des différents types de rayons lumineux de la figure 1 :

1. Rayons ni utiles ni parasites : ce sont ceux qui passent à côté de la boîte et de l'oeil, devant, derrière, audessus, au-dessous. Ils ne rentrent ni dans la boîte ni dans l'oeil. Ils n'atteignent pas le calque et ne diminuent pas le contraste. On peut ranger dans cette catégorie ceux qui frappent la paroi de la chambre par l'extérieur, et qui sont soit ré-émis (diffusion), soit absorbés.

2. Rayons utiles : ce sont ceux qui proviennent des sources de lumière qui éclairent l'objet, qui donnent ensuite naissance à des rayons qui partent de l'objet. Ceux qui ont la chance de passer par le petit trou continuent en ligne droite jusqu'au calque, et participent à la formation de l'image.

3. Rayons parasites, dont nous distinguerons deux types :

3. a. ceux qui rentrent par le trou mais ne frappent pas directement le calque. Ils proviennent des objets hors $d u$ champ. Après une diffusion par la cloison interne, ils peuvent venir frapper le calque dans les parties sombres de l'image et diminuer le contraste. On les élimine par la pose de peinture noire à l'intérieur de la chambre. Il est aussi très efficace de les éliminer avant leur entrée dans la chambre, par la pose d'un pare-soleil, qui est une cloison supplémentaire, extérieure à la chambre.

3. b. ceux qui frappent le calque par l'extérieur de la chambre (du côté de l'observateur). Ils sont souvent 
intenses par rapport aux rayons qui sont passés par l'intérieur de la chambre. Ce sont ceux-là qu'on élimine en disposant un voile opaque autour de la tête de l'observateur.

En conclusion, nous pouvons dire que ce qui est actif dans cet appareil, c'est la cloison de la chambre. Son rôle est de trier les rayons. Ce qui est essentiel est cette séparation entre l'intérieur et l'extérieur. C'est un travail sur l'espace, la limitation d'un lieu par une frontière.

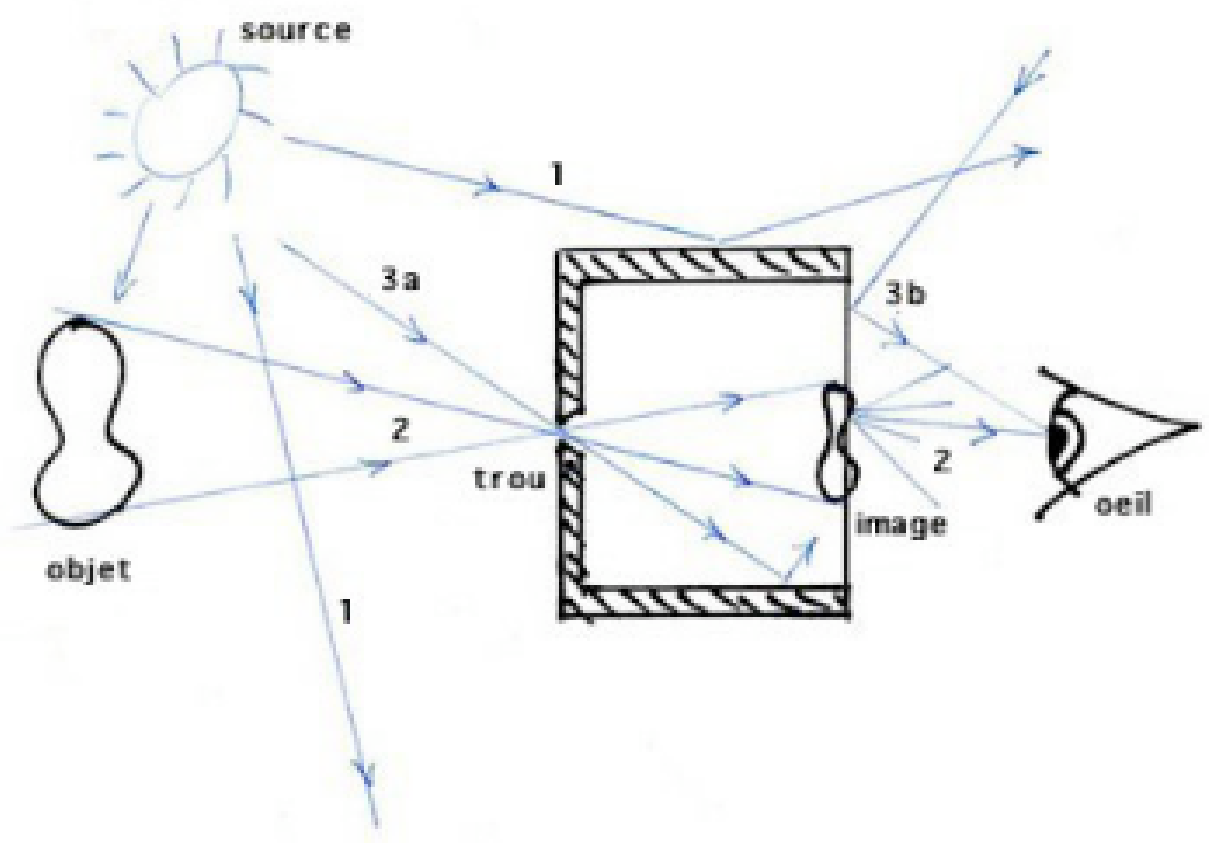

Fig 1 : Camera obscura : machine à trier les rayons lumineux (en coupe)

Pour transformer cette chambre noire en appareil photographique, il suffit d'installer une surface sensible ou capteur à la place du papier calque. Quel que soit la technologie du capteur — daguéréotype, calotype, argentique, numérique, etc. (Frizot, 1994) - il s'agit toujours de transformer l'énergie des photons en énergie électrique. Il faut prévoir une durée d'exposition (que l'on appelle aussi temps de pose), temps nécessaire à l'enregistrement d'un certain nombre de photons, afin qu'après le développement (traitement ultérieur sur le capteur) les parties les plus exposées à la lumière apparaissent blanches et les moins exposées apparaissent noires.

Comparons ce dispositif photographique avec le dispositif représenté par Dürer (figure 2), afin de montrer qu'il s'agit bien de la même représentation, appelée perspective (Lothier, 2004).

$\mathrm{Au}$ milieu de la gravure de Dürer se trouve un cadre transparent quadrillé que nous appellerons le tableau. Nous pouvons considérer que c'est dans ce plan que se forme l'image. Sur la gravure, l'opérateur reporte des points sur un papier qui est quadrillé de la même façon que le tableau, ce quadrillage ne servant qu'à la précision du report. Le travail de l'opérateur consiste à déplacer son oeil afin que chaque position de l'oeil révèle un alignement entre 3 points : un point de l'objet, un point de l'image, le sommet de l'obélisque. Un tel alignement permet de trouver le point image qui correspond au point objet choisi. La répétition de cette opération permet de dessiner l'image point par point. L'oeil de l'observateur n'intervient pas ici pour voir l'objet (d'ailleurs le point de vue n'est pas défini par l'oeil mais par le sommet de l'obélisque), l'oeil intervient pour chercher les alignements. C'est donc un tri des rayons, un par un, chaque rayon matérialisant un alignement qui permet de tracer un seul point de l'image. (C'est identique aux algorithmes modernes de lancer de rayons.)

Lorsque nous comparons l'appareil photographique au dispositif de Dürer, nous pouvons repérer deux différences (figure 3) :

1. Le capteur de Dürer, qui est ce que nous avons appelé tableau, est en avant du point de convergence (le sommet de l'obélisque), alors que pour l'appareil photographique il est en arrière du point de convergence (le 
trou). Néanmoins, nous pouvons aisément vérifier qu'avec la même distance $p$ entre le point de convergence et le plan du capteur, nous obtenons dans les deux cas des images identiques, elles sont simplement disposées symétriquement par rapport au point de vue (le trou de la chambre noire).

2. Dans le dispositif de Dürer, il y a une participation active de l'homme qui dessine car c'est lui qui trie les rayons, un par un. Il n'y a pas la cloison (de la chambre) pour les trier automatiquement. Et il n'y a pas le capteur photo qui enregistre automatiquement.

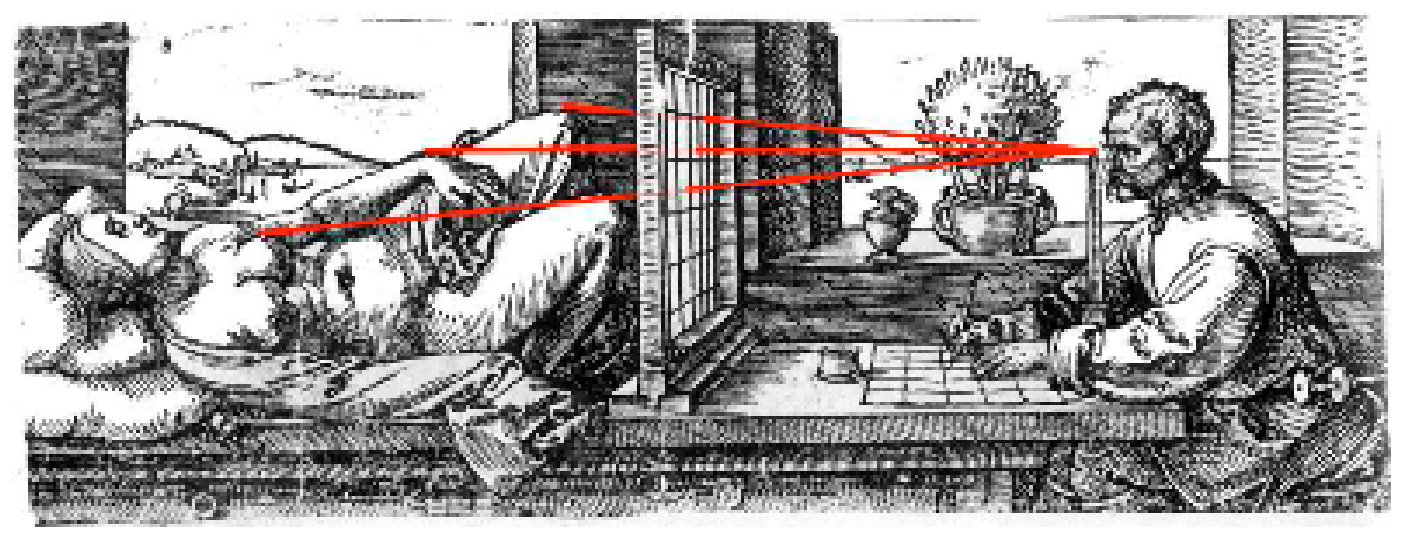

Fig 2 : Perspective de Dürer
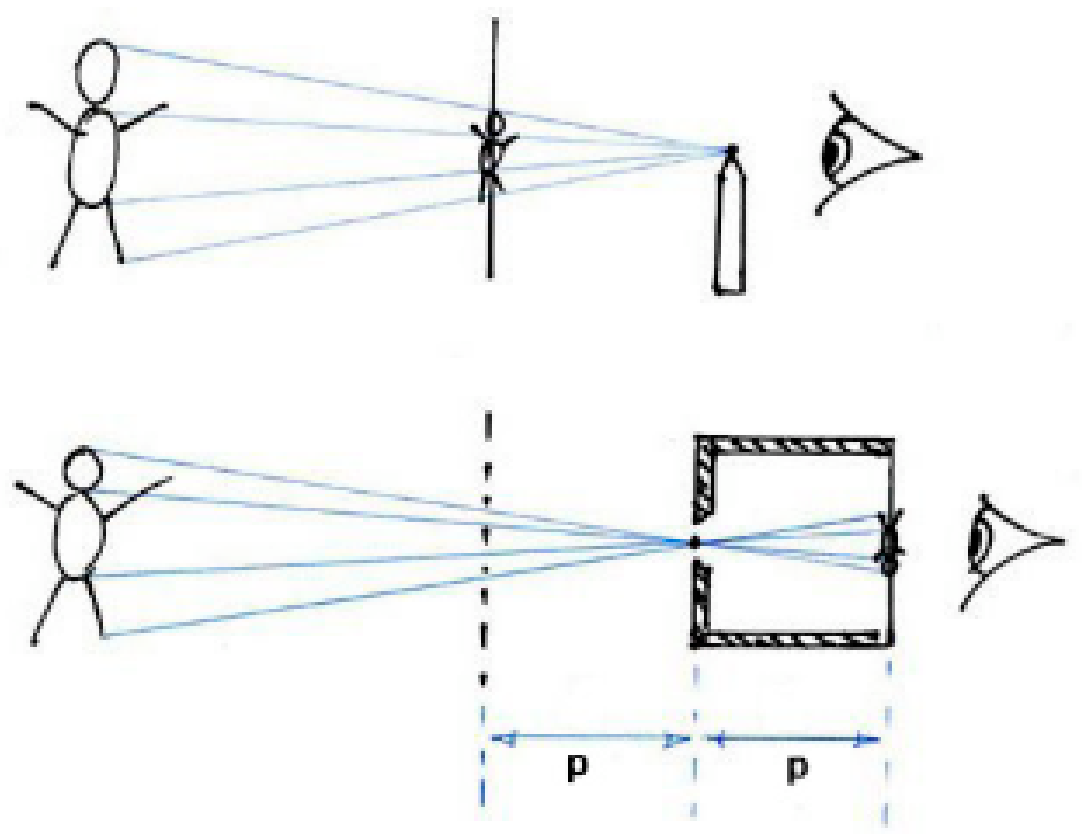

Fig 3 : Comparaison des deux perspectives

On peut dire, pour reprendre des termes de l'informatique, que le dessinateur de Dürer trie les rayons successivement, en séquentiel, alors que la cloison de la chambre trie les rayons simultanément, en parallèle. Vue ainsi, l'automaticité de l'appareil photographique serait double, d'une part pour le tri des rayons, d'autre part pour l'enregistrement par le capteur. L'automaticité du tri par la paroi nous semble au moins aussi importante que celle du capteur. 
L'image obtenue dans la photographie par sténopé est donc identique à celle obtenue par la perspective de Dürer. Lorsque nous remplacerons le trou du sténopé par des lentilles de verre, la géométrie de l'image (nous l'appellerons perspective) sera également identique. (C'est ce que l'on appelle le centre optique des lentilles qui jouera le rôle du point de vue.)

Dans les deux dispositifs que nous venons de décrire, le point de vue n'est pas défini par la position de l'oeil, mais il est défini par un objet matériel particulier, sommet de l'obélisque chez Dürer, sténopé pour l'appareil photographique. Par ailleurs, l'image n'est pas mentale, c'est un objet matériel (en optique, on parle d'image réelle). L'objet, l'image et le point de vue sont donc tous les trois des objets matériels.

À l'aide de ce simple modèle d'appareil photo sténopé, nous pouvons retrouver les explications de (presque) toutes les caractéristiques des appareils photos : perspective, angle de champ, rapport de réduction, ouverture, temps de pose. Passons rapidement ces points en revue.

\section{2. Angle de champ et rapport de réduction}

Faisons varier la profondeur de la chambre. Considérons deux chambres dont la seule différence est la profondeur, placées à la même distance du même objet. La construction des images suivant les figures précédentes nous montre immédiatement : la chambre la plus profonde donne une image plus grande, la chambre la moins profonde donne un champ plus grand. Ceci est l'effet connu d'un zoom, c'est un effet direct de la perspective. Le paramètre de l'appareil qui influence la taille de l'image (et donc le rapport de réduction) est la profondeur de la chambre. C'est un étalon auquel on compare le monde.

\section{3. Ouverture et temps de pose}

Soit un trou-sténopé de diamètre $d$. Soit $p$ la profondeur de la chambre. Appelons ouverture ou $O$ le rapport $p / d$. La quantité de lumière (en nombre de photons) reçue par un élément d'aire du capteur est proportionnelle au temps d'ouverture du trou $t$ (temps de pose) et inversement proportionnelle au carré de $O$. Pour un appareil photographique, ces deux nombres $t$ et $O$ conditionnent la bonne exposition du capteur. L'ouverture $O$ est le nombre qui est gravé à côté du trou (sur l'objectif s'il y a des lentilles).

\section{4. Lentilles de verre, réglage de la distance}

Sans avoir parlé de lentille de verre, mais uniquement de sténopés, nous avons introduit presque toutes les notions nécessaires à la compréhension de l'appareil-photographique : perspective, taille de l'image, angle de champ, temps de pose, ouverture. Il ne manque que l'introduction de : distance de mise au point, profondeur de champ. Pour ces deux derniers, il faut impérativement parler de lentilles de verre, ou plutôt, et plus généralement, de systèmes faisant converger les rayons lumineux.

Avec un sténopé de petit diamètre, les temps de pose sont en général trop longs. On va donc chercher à augmenter le diamètre du trou, mais l'utilisation de sténopés de fort diamètre conduit à des images trop floues, à moins qu'on trouve un moyen pour faire converger les rayons lumineux au moment de leur franchissement du trou (figure 4).

Ceci est obtenu avec une lentille de verre ou une combinaison de lentilles de verre, que l'on nomme objectif en photographie. Mais ceci amène un phénomène nouveau : Si les rayons convergent en $A^{\prime}$, ils ne convergent pas en un autre point $A^{\prime \prime}$. Pour obtenir une image nette, le capteur ne peut pas être situé à n'importe quelle distance du trou, comme c'était le cas avec le sténopé. On dit que $A$ et $A^{\prime}$ sont des points conjugués. Un objet en volume (ayant une épaisseur) donne une image en volume à l'intérieur de la chambre. Comme le capteur est plan, le photographe ne peut pas capter cette image en volume, il en est réduit à ne pouvoir conserver une image nette que pour un seul plan. Il doit donc choisir la position du capteur, ce qui entraîne un choix de la partie de l'objet qui sera représentée avec netteté. C'est la notion de mise au point ou réglage de la distance à l'objet.

Le point conjugué de l'infini sur l'axe optique (photographie d'un astre du ciel par exemple) est appelé foyer. Pour cette raison, un tel système optique, qui fait apparaître des points conjugués, est appelé système focal. La distance entre un des foyers et la lentille (supposée d'épaisseur négligeable) est nommée distance focale.

Il n'est nul besoin d'étudier de près les lentilles et leur fonctionnement pour établir les formules qui régissent la mise au point. Tous les cas de construction d'image se déduisent de la position des foyers par des raisonnements de géométrie élémentaire. En conséquence, tout système focal, quel que soit sa nature, qui assure une convergence de quelque rayons ou particules que ce soit (pensons par exemple aux microscopes électroniques ou aux lentilles gravitationnelles), obéïra aux mêmes lois, représentées par les mêmes formules mathématiques. 
Ces formules sont donc déduites uniquement de la propagation en ligne droite et de l'existence de couples de points conjugués.
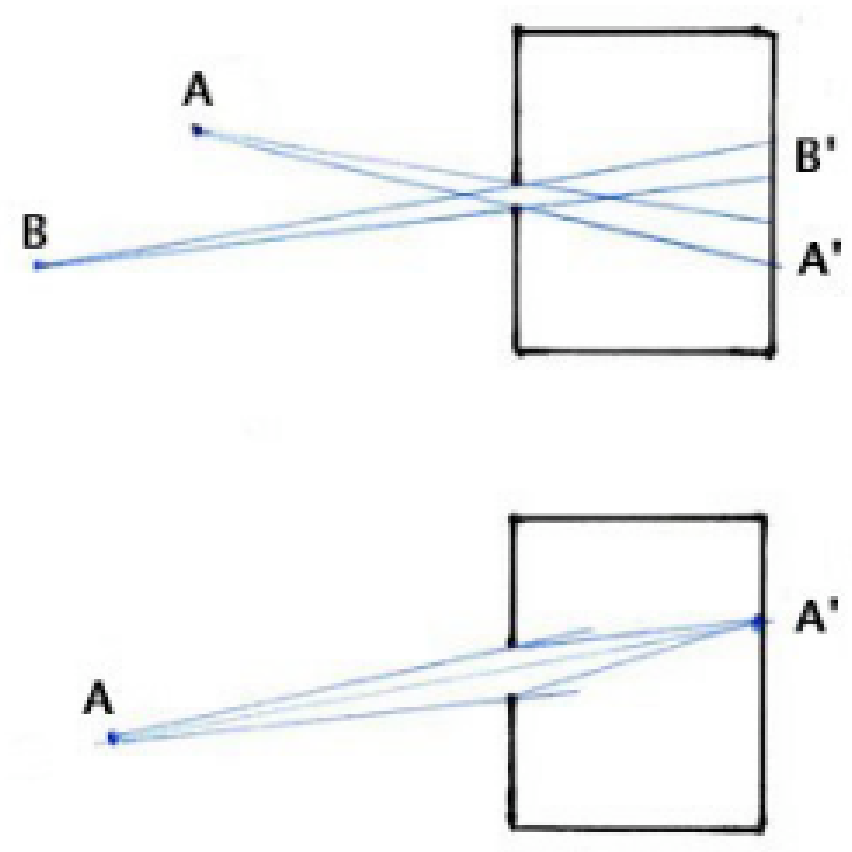

Fig 4 : Lentille-objectif

\section{5. Profondeur de champ}

Nous ne détaillerons pas cette section, et demandons au lecteur intéressé de se reporter à notre étude de (Matherat, 2006). Là aussi, il ne s'agit que de géométrie élémentaire autorisée par la propagation de la lumière en ligne droite. Nous rappellerons uniquement le résultat essentiel : dans tous les cas où la profondeur de champ n'est pas très grande, elle ne dépend que du rapport de réduction, qui lui ne dépend que du format du capteur (à cadrage égal).

Ainsi, en inversant cette proposition, nous pouvons être conduits à nous poser la question suivante : En regardant une photo, peut-on en déduire le format de l'image originale? (Non la taille de l'image reproduite sur un support imprimé, mais la taille du capteur.) Il se trouve que dans beaucoup de cas la réponse est positive. C'est assez surprenant, mais nous avons pu le montrer sur des exemples. Dans tous les cas ou l'objet n'est pas trop grand devant la taille de la chambre (c'est-à-dire dans les cas où la profondeur de champ n'est pas trop grande), il est possible de deviner la taille de la chambre en regardant la photographie.

Comme il est reconnu que la profondeur de champ est un paramètre important parmi les possibilités d'expression du photographe, on doit admettre que ce dernier sera amené à changer de chambre afin de changer le format du capteur. En photographie numérique, on dispose plutôt de petits formats de capteurs. Sinon, c'est très cher et/ou très contraignant. On a donc en général de très grandes profondeurs de champ comparativement aux chambres plus grandes accessibles en argentique.

\section{6. Conclusion de cette première partie}

Nous avons passé en revue les différents paramètres de prise de vue d'un appareil photographique, et nous avons retrouvé toute l'optique photographique en faisant très peu de calculs, en utilisant simplement quelques résultats de géométrie élémentaire. Nous avons montré ainsi que tout ce qui concerne la formation de l'image (la perspective), et son enregistrement sur un capteur, peut être déduit de la proposition la lumière se propage en 
ligne droite.

De tous les paramètres de prise de vue d'un appareil photographique, seules la distance de mise au point et la profondeur de champ sont étroitement liées à l'utilisation d'un système focal. Et encore, il n'est nullement nécessaire de préciser la nature des lentilles (ni même l'existence de lentilles), il suffit de signaler l'existence de couples de points conjugués, ce qui est requis par la volonté d'obtenir des images nettes avec des trous plus grands que des trous d'aiguille.

Notre façon d'exposer cette optique a mis en évidence le rôle essentiel joué par la cloison de la chambre noire et ses dimensions : 1) cette cloison trie les rayons et forme ainsi l'image de manière automatique ; 2) la profondeur de la chambre définit la taille de l'image et se comporte donc comme une unité de mesure du monde ; 3) la largeur du capteur détermine la profondeur de champ pour un système focal. À coté de ces trois fonctions essentielles de la boîte, les rôles de l'objectif et du capteur paraissent bien secondaires.

La seule remarque que nous ayons faite à propos de l'apparition des capteurs numériques concerne le fait que ces capteurs sont généralement petits, ce qui conduit à de grandes profondeurs de champ. De cette première partie, nous pouvons déduire que nous n'avons pas rencontré le virtuel dans cette exploration des appareils photographiques numériques, ou en tous cas nous n'avons pas trouvé quelle signification donnée à ce mot pourrait être reliée à ces nouveaux capteurs. Cherchons alors le virtuel autrement : par la voie d'une interrogation de la notion de représentation, dans son rapport avec la vision.

\section{QU'EST-CE QUE VOIR ?}

\section{1. Introduction}

Dans cette deuxième partie, nous allons saisir l'opportunité d'un regard critique sur la première partie. Nous avons mis en évidence la trame du discours de l'opticien, nous pouvons nous demander ce que ce discours nous apprend sur le réel. Nous verrons que cela nous conduira à une formulation de ce qu'est voir, et nous pourrons la mettre en parallèle avec ce qu'est mesurer pour l'expérimentateur, et ce qu'est simuler pour l'informaticien qui produit des images de synthèse. Nous serons ainsi conduit à approcher une notion de virtuel qui pourrait être celle à laquelle on semble faire allusion dans le contexte des images numériques.

Dans la première partie, deux points nous semblent en particulier soulever des questions :

1. Tous les raisonnements précédents s'appuient sur la proposition : la lumière se propage en ligne droite. Quel est le statut logique de cette proposition ? S'agit-il d'une découverte du physicien qui a observé la nature de la lumière, ou bien s'agit-il au contraire d'un principe qui permet de construire l'espace et les objets ? On ne peut accepter de considérer qu'il s'agit d'une vérité (sur la nature), qui serait indépendante de la construction du discours des hommes. Le rôle de cette proposition dans le discours est un point à éclaircir.

2. Dans les figures de l'exposé précédent, nous avons dessiné un oeil. Il s'agit d'une représentation d'un sujet de la perspective. Dans la figure qui représente la chambre avec un calque, l'oeil est dessiné derrière le calque, cela nous dit pour qui l'image prend un sens. Dans la gravure de Dürer, nous avons vu que le dessinateur prend une part active au tri des rayons, il déplace son oeil pour trouver les alignements, ce sujet montre ainsi de façon encore plus évidente son intention. Dans les figures qui ont été faites ensuite, l'oeil devient implicite car le capteur photographique est opaque, mais il y a toujours le sujet qui met en place l'expérience et qui ensuite regarde la photographie.

On oublie en général qu'il y a un deuxième sujet, et qui n'est pas dans la même posture, il s'agit de celui qui regarde ces schémas : par exemple le physicien qui énonce le cours de physique. Ce deuxième sujet se place en dehors du plan du schéma et regarde comment fonctionne la perspective pour le premier sujet.

Ces deux sujets peuvent-ils être confondus (ou tout du moins leurs rôles, leurs fonctions)? Pour le physicien, il est important qu'il n'y ait pas de distinction entre les conceptions (visions du monde ?) de ces deux sujets, car il est posé en principe que les lois de la physique sont universelles et objectives. Dans ce contexte, ces lois doivent s'appliquer aussi bien au premier sujet, qui est transformé pour l'occasion en objet dans l'énoncé $\mathrm{du}$ second sujet. Du coup, elles doivent s'appliquer aussi au deuxième sujet, qui ne doit pas pouvoir être distingué du premier.

On remarquera que cette attitude du physicien est celle qui est implicitement adoptée par Dürer puisque son schéma de construction de la perspective est lui-même dessiné en perspective. Il suppose donc au sujet qui énonce le cours de perspective la même façon de voir que celle du sujet qu'il dessine comme dessinateur. 
Si on adopte cette attitude, lorsque le deuxième sujet regarde la représentation du premier en train de voir, il pose que sa propre vision est identique, et donc il se voit en train de voir.

\section{2. Réalité, raison et métaphysique}

La lumière se propage en ligne droite. Quel est le statut logique de cette proposition?

— Si on sait déjà ce qu'est une ligne droite, cela nous informe sur une propriété de la lumière. Mais est-ce :

1) une définition de la lumière?

2) une connaissance acquise par l'observation de la lumière?

3) une propriété démontrée à partir d'une autre définition de la lumière ?

- Si on ne connait pas la ligne droite,

4) on peut utiliser la propagation de la lumière pour définir la ligne droite.

Cela nous donne accès à quatre statuts logiques différents pour cette proposition. Nous ne prétendons pas qu'il n'y en ait que quatre, on peut probablement en trouver d'autres.

Donnons des exemples pour illustrer ce propos. Le menuisier, pour vérifier la rectitude de son tasseau, le regarde en bout pour voir si l'arête se voit comme un point. Il utilise donc la lumière comme définition de la ligne droite. Un jardinier plante des piquets. Il veut que ses piquets soient alignés (ligne droite), alors il se place en bout et regarde. Je veux ne voir qu'une tête. Si les piquets se cachent les uns derrière les autres, alors ils sont alignés. Ici encore, il s'agit d'utiliser la lumière comme définition de la ligne droite. C'est une méthode très largement utilisée dans la pratique. Pour la construction de bâtiments et les travaux de terrassement, on utilise des lunettes de visée ou des faisceaux laser. Dans tous ces cas, la proposition ne peut rien nous apprendre sur la propagation de la lumière.

Mais plaçons-nous par hypothèse dans la situation où on aurait pour la ligne droite plusieurs définitions possibles. On pourrait se trouver dans la configuration suivante (figure 5) qui représente un monde plan avec ses habitants (dans le plan de la figure) alors que nous nous plaçons en dehors de ce monde plan (avec des lois différentes).

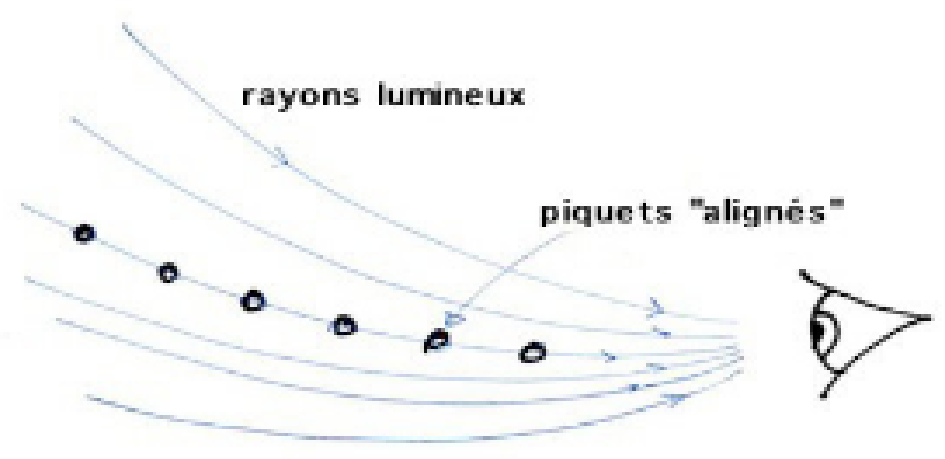

Fig 5 : Situation de caverne platonicienne

L'oeil dessiné verrait que tous les piquets se cachent derrière le premier (propagation de la lumière), or nous avons dessiné des rayons courbes. Il se pourrait qu'aucun sujet du monde du plan ne se doute que la lumière ne se propage pas en ligne droite, connaissance que nous aurions nous, nous qui ne sommes pas du même monde et qui pouvons dire : ces gens croient que la lumière se propage en ligne droite.

C'est une autre version de la caverne de Platon (La république, livre VII), et c'est un moyen d'introduire une question metaphysique. Platon l'utilise pour dire que les esclaves (ceux qui restent dans le plan du dessin) ne voient que les apparences alors que les êtres intelligents accèdent à l'essence des choses. Mais nous pourrions tout aussi bien l'utiliser pour dire au contraire que si certains sujets ont moins de connaissances que nous sur la réalité, alors on peut être amené à se demander si notre propre connaissance n'est pas elle aussi limitée, si des êtres plus intelligents ne pourraient se moquer de nos insuffisances.

Renonçons à accéder à l'essence des choses. Par contre, considérons que les apparences des choses changent suivant le langage utilisé pour bâtir des expériences sur ces choses. Ce que nous pouvons apprendre du monde extérieur en faisant des expériences de physique dépend de la structure du discours (Kant, 1783). Donnons un 
exemple pour illustrer ce point.

Nous connaissons une autre méthode pratique pour aligner des piquets : le cordeau. On tend un fil, et on dit qu'il réalise une ligne droite. C'est une deuxième définition pour la ligne droite qui consiste à dire : tendre, c'est trouver le plus court chemin, et c'est une définition de la ligne droite. Dans le plan précédent, le fil tendu seraitil vu droit par ses habitants? (figure 6).
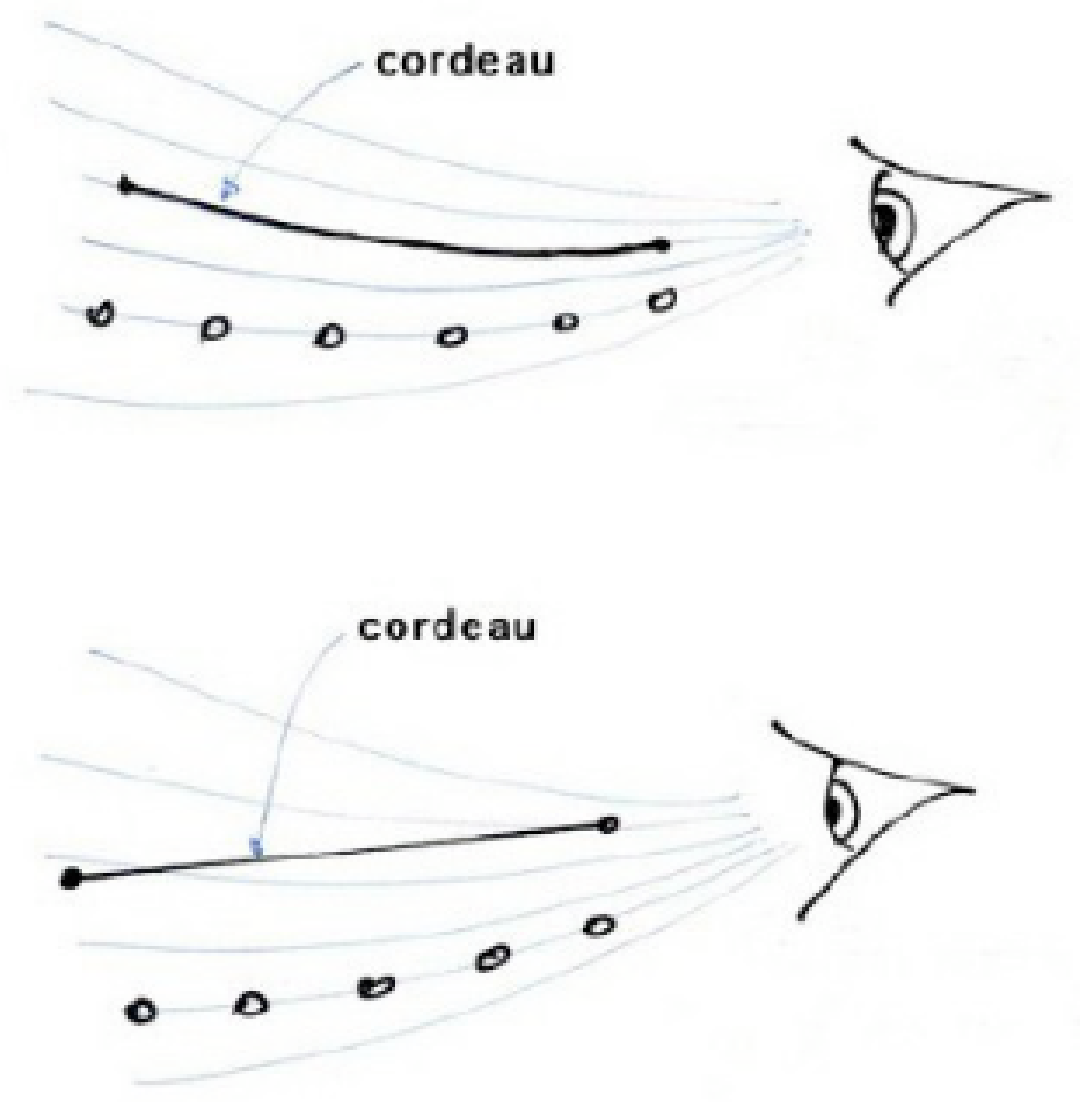

Fig 6 : Le cordeau est-il comme les rayons lumineux ?

Dans cette figure, il y a deux possibilités pour chacun des deux cas représentés :

1. Si on définit la ligne droite par la lumière, alors, dans la situation du haut, les piquets et le cordeau sont droits. Cela ne nous apprend rien sur la lumière. Dans la situation du bas, les piquets sont alignés, mais le cordeau n'est pas droit (car il n'est pas comme les rayons lumineux). Cet écart entre le cordeau et les piquets nous apprend quelque chose sur le cordeau (nous apprenons que le plus court chemin n'est pas la ligne droite).

2. Si on définit la ligne droite par le cordeau, alors dans la situation du haut, la lumière et les piquets sont droits (car ils sont comme le cordeau) et on apprend que la lumière se propage en ligne droite (ce qui est alors une connaissance acquise par l'expérience). Dans la situation du bas, ni la lumière ni les piquets ne sont droits (car ils ne sont pas comme le cordeau), et on apprend là aussi quelquechose sur la lumière (on apprend qu'elle ne se propage pas en ligne droite).

En conclusion, le fait qu'on puisse ou non apprendre quelque chose du monde extérieur dans une expérience de physique, ainsi que ce qu'on peut y apprendre, dépend du système logique dans lequel on se place. Cela dépend, non seulement des règles de déduction (c'est-à-dire de la mécanique du raisonnement), mais aussi des choix des postulats et des définitions, c'est-à-dire plus généralement du langage logique. En d'autres termes, la réalité (tout du moins ce qu'on en connaît et ce que l'on peut en dire) dépend du langage.

Ce que disent les physiciens, ou plutôt ce qu'ils disaient avant la relativité (Einstein, 1905), c'est que dans l'espace 3D euclidien où nous vivons, la lumière se propage suivant le même chemin que le fil tendu. Le plus court chemin n'a de sens que dans un espace où il existe une notion de distance indépendante de l'observateur. 
Les mathématiciens disent que ce n'est pas vrai de tous les espaces, mais que c'est vrai dans les espaces euclidiens. Par ailleurs, le fait que le trajet de la lumière soit le plus court chemin est connu en physique sous le nom de principe de Fermat (si l'on entend plus court dans le temps), qui est une façon possible de fonder l'optique (Feynman, 1963).

Mais tout cela, ce ne sont pas des choses qui sont données, qui nous tombent dessus, envoyées par des essences qui pré-existent au langage. Il a fallu inventer tous ces concepts de ligne droite, lumière, plus court chemin, euclidien, etc... et il a fallu trouver un discours cohérent pour relier ces concepts, et le relier à des expériences.

\section{3. Les sens sont de contact}

Notre connaissance du monde extérieur dépend aussi de nos sensations, c'est-à-dire de ce que font nos sens. De ces possibilités découleront des contraintes qui conditionneront les expériences imaginables par le physicien et leurs interprétations. Un point essentiel réside dans le fait suivant : La vue est une sensation de contact : l'oeil ne sent pas à distance. Ce n'est pas propre à la vue, c'est le cas de tous les sens : toucher, goût, odorat, ouïe, vue. (Remarquons une difficulté : le verbe sentir en français est plutôt associé à l'odorat, et nous ne disposons pas d'un verbe plus général associé à tous les sens. Nous avons pourtant besoin, dans ce qui suit, d'un tel verbe pour le sens de la vue. Nous utiliserons tout de même sentir, en le pensant en relation avec sens et sensation.)

1. Le toucher est, bien entendu, un sens de contact. Il faut un contact entre l'objet et la peau pour sentir par le toucher. C'est une sensation à la surface de notre corps.

2. Le goût est aussi un sens de contact, localisé sur la langue, donc plus intime puisque un peu intérieur, au contact de muqueuses. Mais si on dit que ces muqueuses sont une partie de la frontière entre notre corps et le monde extérieur, alors la situation est semblable à celle du toucher.

3. Pour l'odorat, c'est un peu plus compliqué car on peut avoir l'impression de sentir à distance, par exemple l'odeur d'une fleur située à un mètre devant nous. Les physiologistes nous disent que des molécules de vapeur du parfum sont venues se dissoudre à la surface de notre muqueuse nasale. Il s'agit là encore d'un contact intime à la frontière de notre corps. Il y a eu propagation de matière dans l'air. C'est une partie de la matière de la fleur qui est venue jusqu'à nos muqueuses. Cette notion de propagation n'est pas évidente, elle a été construite, à partir des notions d'espace et de temps. Elle est compatible avec l'idée que notre corps occupe un lieu de l'espace, qu'il est limité par une frontière, et qu'il a un extérieur. Dans cet espace extérieur, il peut y avoir propagation, c'est-à-dire changement de lieu qui prend du temps.

4. Pour l'ouïe, c'est un peu la même chose, mais plus troublant encore car nous avons aussi l'impression d'entendre à distance, à une distance encore plus grande, et la propagation est plus rapide. Des expériences nous montrent que le son ne se propage pas sans l'air, qu'il est une perturbation de la pression de l'air, perturbation qui se propage. L'oreille ne sent que les variations de pression localisées sur le tympan, et non à distance. Cela permet de la tromper si nous portons des écouteurs qui créent une illusion sonore. Ces expériences d'illusions prouvent que l'ouïe est aussi une sensation de contact, à la frontière de notre corps.

5. Pour la vue, on ne sent que les rayons lumineux qui entrent dans notre oeil, et non ceux qui passent devant sans le rencontrer. L'oeil ne peut sentir non plus comment se propagent les rayons lumineux avant qu'ils n'atteignent notre oeil, il ne peut donc savoir s'ils se propagent en ligne droite. Ce n'est ni une propagation de matière (comme pour l'odeur), ni une propagation de déformation d'un milieu (comme pour le son), puisque la lumière se propage aussi dans le vide. Ce que l'oeil sent, c'est la direction des rayons lumineux entrants. Le couple (cristallin, rétine) permet une discrimination de direction (locale, en le lieu de l'oeil) des rayons. Cette conception est compatible avec les expériences d'illusion optique, analogues des illusions sonores obtenues avec les écouteurs. La plus classique de ces illusions est celle liée à l'utilisation du miroir (figure 7).

Si l'observateur voit la table virtuelle derrière le miroir, c'est bien qu'il ne sent pas à distance, sinon il sentirait qu'il n'y a pas de table à cet endroit. Si l'oeil sentait à distance, on verrait à travers le miroir, et le miroir n'aurait aucun effet sur nous, ce ne serait pas un miroir. Aucune expérience d'illusion optique ne fonctionnerait. Il n'y aurait pas non plus de corps opaque. On ne verrait donc aucun objet !

Si nous voulons conserver la consistance de l'ensemble des connaissances que les physiciens rassemblent sous le nom d'optique, il est essentiel de conserver la notion de propagation de la lumière. Cela conduit à considérer que l'oeil ne sent que la direction des rayons lumineux entrants, et qu'il ne peut sentir à distance. Ce n'est donc pas l'oeil qui peut voir, ou en tous cas ce n'est pas l'oeil seul qui peut voir, si on appelle voir ce qui consiste en : construire une connaissance visuelle de l'espace et des objets.

On a donc mis là au jour une confusion de langage dans laquelle nous sommes tous pris : il nous faudrait 
deux verbes différents pour nommer d'une part l'action de l'oeil, ce que fait l'oeil, sa fonction, et d'autre part l'action de construire l'espace et les objets, l'action de connaître ce dans quoi se trouvent les objets et notre corps.

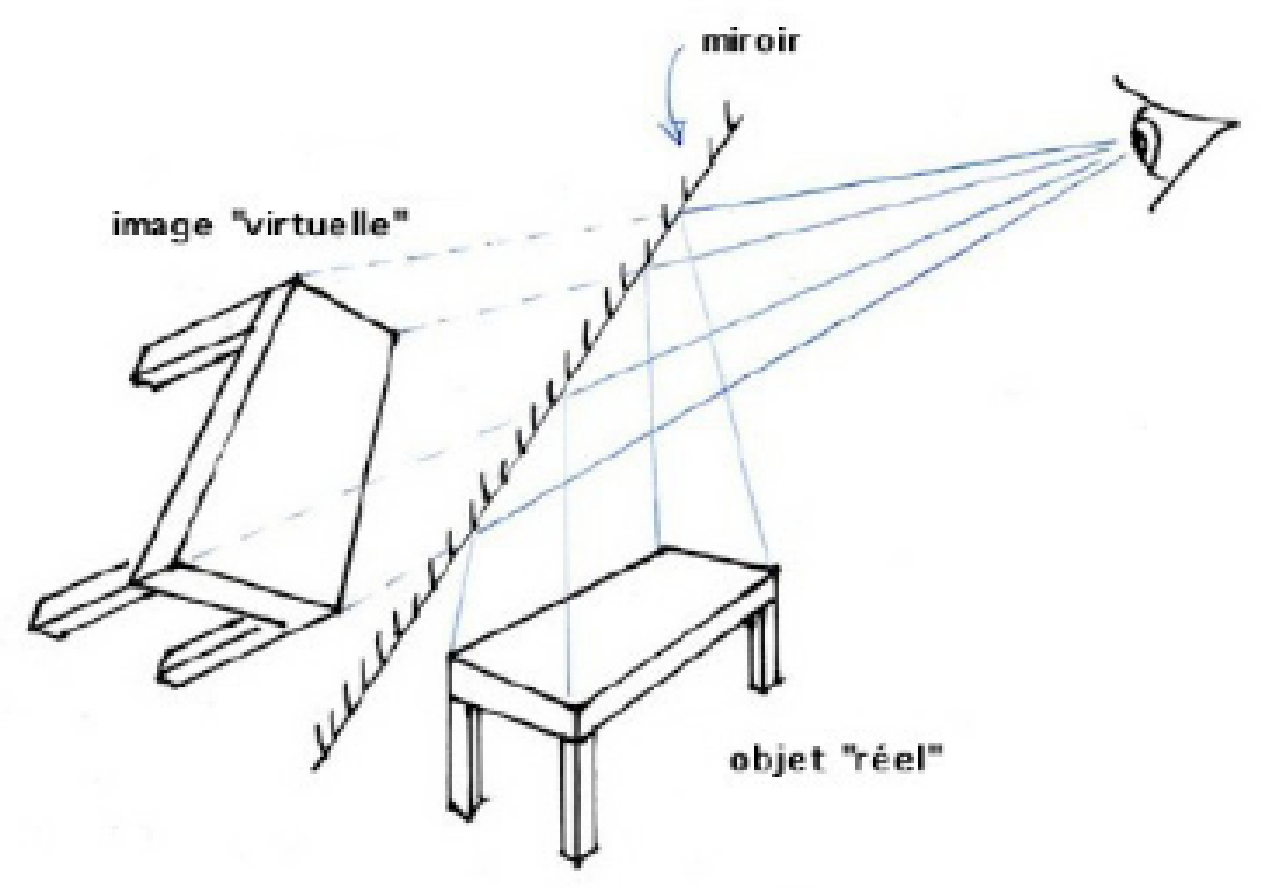

Fig 7 : Le miroir nous montre que l'oeil ne sent pas à distance

Pour la suite de cet exposé, nous ferons le choix d'utiliser les mots sensation et sentir pour désigner ce qui est de contact, c'est-à-dire le phénomène local à la surface du corps, en particulier ce que fait l'oeil. Et nous utiliserons les mots vision, voir ainsi que perception, percevoir pour désigner la construction de la connaissance (visuelle) de l'espace et des objets.

(Nous réserverons un sort particulier au mot vue que nous éviterons d'utiliser, car il devrait être associé à voir, alors que nous avons déjà été obligé de l'utiliser pour le sens de la vue. Il est bien difficile de faire autrement en raison de la confusion constante dans le langage courant.)

Avec ces définitions, le voir ne sera pas confondu avec une sensation. L'oeil ne sentira pas à distance, mais l'homme verra, percevra à distance. L'oeil, étant lié uniquement à la sensation, ne sera donc pas seul l'organe du voir.

\section{4. Comment voyons nous à distance?}

Notre oeil ne sent que la direction locale des rayons lumineux entrants (notons que c'est le seul point d'analogie que nous ferons avec la camera obscura), et non leur direction à quelque distance de là. Ainsi, si ces rayons ne sont pas des lignes droites, peut-être sommes-nous toujours dans une situation de mirage, situation analogue à celle du miroir. La table que je crois voir devant moi pourrait être à situer dans une autre direction.

Comment peut-on savoir si la lumière se propage en ligne droite ? On ne peut pas voir avec notre oeil la lumière se propager, puisque ce que sent l'oeil est un rayon qui ne se propage plus. Pour observer comment la lumière se propage, il faudrait observer le même rayon lumineux à plusieurs endroits de son parcours, ce qui est impossible puisqu'un photon observé est un photon absorbé. On en est donc réduit à obtenir cette connaissance de façon indirecte, par exemple par les expériences d'optique. Mais si l'optique géométrique est construite en supposant que la lumière se propage en ligne droite, alors nous sommes pris dans une boucle.

Comment faire? De toutes façons, nous devons construire le monde extérieur à partir de nos sensations de contact. Donc nous devons extrapoler le monde extérieur à partir de nos sensations. 
Une attitude possible consiste à postuler que la lumière se propage en ligne droite (cela suppose que nous ayons une autre définition de la ligne droite : par exemple espace euclidien et plus court chemin). La direction d'arrivée d'un rayon dans notre oeil nous donne alors une information sur la direction de l'espace dans laquelle se trouve l'objet : l'objet est quelquepart sur la droite qui part de notre oeil dans cette direction. Ce que nous avons à faire est donc l'inverse de la perspective : en considérant les rayons qui arrivent dans notre oeil, retrouver d'où ils peuvent venir. Par ailleurs, toutes les expériences d'optique viennent conforter la cohérence de ce discours, qui est non pas une vérité sur l'essence des choses, mais la cohérence logique (c'est-à-dire la noncontradiction de l'édifice rationnel).

La proposition la lumière se propage en ligne droite serait donc, dans cette attitude, un postulat, qui aurait la qualité de ne pas être en contradiction avec l'expérience.

En relation avec cette attitude, nous pouvons remarquer l'importance de l'expérience du miroir, car le miroir ne peut être vu que si l'on se voit en train de voir. En effet, pour comprendre ce que fait un miroir, il faut se dessiner la figure 7, dans laquelle on représente : l'objet, le miroir, le sujet, les rayons lumineux, ainsi que les angles de réflexion des rayons sur le miroir (lois de Descartes).

C'est seulement en ayant la figure 7 présente à l'esprit qu'on peut voir le miroir lui-même. Sinon on ne peut avoir conscience que ce qu'on voit est une image virtuelle symétrique de l'objet réel. On ne peut pas voir sans comprendre. Bien sûr, on peut voir autre chose si on comprend autre chose, mais cet autre chose sera-t-il lui aussi cohérent avec le reste ? On peut se trouver face à une situation paradoxale, un conflit de significations. En général, ce conflit se détecte sur les bords, le cadre du miroir. Face à un miroir infini, nous n'aurions aucun moyen d'affirmer que c'est un miroir, nous serions obligé de conclure que l'espace lui-même est symétrique, et que l'on se cogne en essayant de passer le plan de symétrie. Si en revanche nous avons la possibilité de passer derrière le miroir et d'interpréter ce que nous voyons sur le bord du miroir, nous pouvons dire : « telle partie de ce que je vois est compatible avec une vision directe, telle autre partie est compatible avec une vision réfléchie, et cela est compatible avec un miroir plan de telle épaisseur dont la limite serait ici, etc. ».

En conséquence, quand on regarde un miroir (et qu'on le voit, c'est-à-dire qu'on le comprend), alors on voit la vision, c'est-à-dire qu'on voit les conventions, plus ou moins explicites, choisies par les hommes pour extrapoler le monde extérieur à partir des sensations de contact. On se voit ainsi participer à cette société construite par ces conventions sur l'espace, qui sont en relation avec le langage des hommes, et l'organisation de leurs connaissances. Tout cela est-il conscient pour tous? Les autres voient-ils la même chose que moi ? Les physiciens voient-ils mieux que les personnes non-éduqués à l'optique ? Les peuples sans miroir ont-ils les mêmes conceptions de l'espace ? Le partage de mêmes conceptions à propos de ces questions ne peut se faire que par l'échange dans le langage, et sont donc tributaires, entre autres, des structures du langage. Voir est un fait culturel.

\section{5. Qu'est-ce qu'une mesure?}

Qu'est-ce qu'une mesure en physique ? Prenons l'exemple d'une mesure de longueur, la mesure de la longueur de la table avec un mètre ruban (figure 8).

On construit un autre objet (le mètre lui-même, en le déroulant), dont on connaît la longueur par construction. C'est sa fonction : le mètre à ruban nous permet de construire un segment de droite de longueur arbitraire, par exemple ici $63 \mathrm{~cm}$. Si les deux objets, la table et le mètre, ont une longueur indistingable, alors on dira que la longueur de la table est égale à $63 \mathrm{~cm}$. On dit qu'on a mesuré la table. Cela ne veut pas dire qu'on a prélevé à la table une information sur elle (il est amusant de remarquer qu'en français le mot perception a une double signification, celle liée aux sensations et celle liée au fisc, qui effectue des prélèvements obligatoires). La seule chose qu'on a fait avec la table est la comparaison indistingable de longueur avec l'autre objet, le mètre, qui est, lui, construit, et prétendument connu par cette construction. Ses graduations résultent d'une comparaison avec une longueur étalon.

Une mesure est la construction d'un autre objet que l'objet mesuré. Le nombre qui en résulte ne vient pas de l'objet mesuré, mais de l'objet autre. C'est la comparaison d'indistingabilité qui nous permet de transférer le nombre, depuis l'autre vers le mesuré. On n'a pas besoin de savoir ce que sont (dans leur essence) : l'espace, les objets, la longueur pour faire cette comparaison.

Précisons ce que nous appelons comparaison d'indistingabilité. Pour cette mesure de longueur, il s'agit de vérification aux deux extrémités (et non tout au long de la longueur) : à chaque extrémité, on doit ne pas pouvoir dire que le lieu n'est pas le même, sinon on ajuste, on déplace. Il ne s'agit pas d'une égalité de lieu ni d'une égalité de longueur, mais il s'agit de non-distinction d'inégalité (deux négations), soit à l'oeil nu, soit avec l'aide d'un appareil. La précision de l'appareil de comparaison définit ce qu'on appelle incertitude de la mesure. 


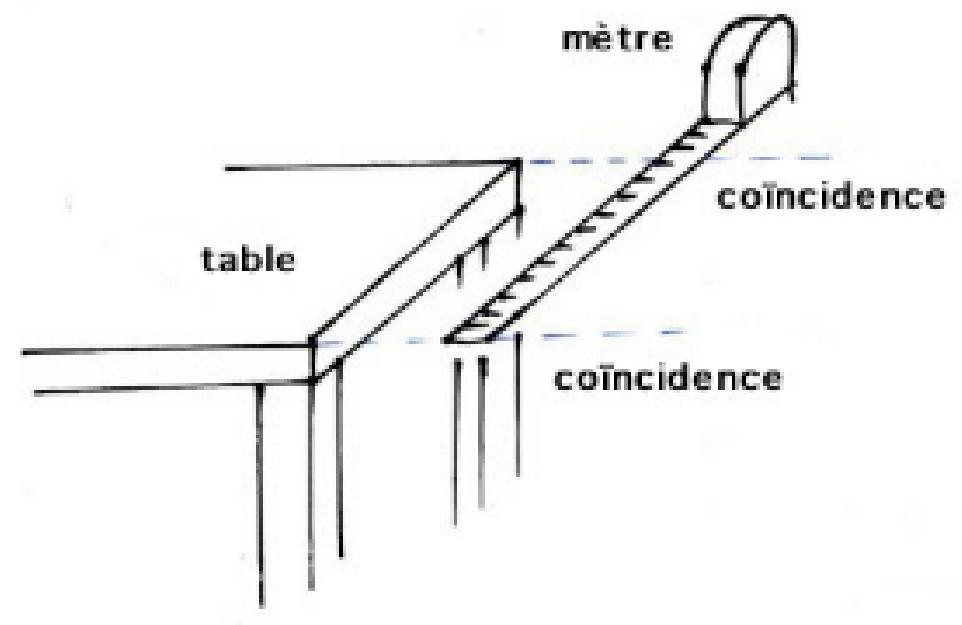

Fig 8 : Mesure de la longueur de la table

Prenons un autre exemple, la balance de Roberval (figure 9).

Ici aussi, on construit un autre objet. Comme il s'agit d'une mesure de masse, on construit une masse connue et on a besoin d'une comparaison d'indistingabilité de masses. La balance n'est que l'instrument de comparaison. Si on ne connaît rien ni de l'essence de la farine, ni de l'essence de la masse, on peut quand même mesurer la masse de la farine par l'intermédiaire de la connaissance de la masse fabriquée. La masse fabriquée est constituée d'addition de masses marquées construites par comparaison à une masse étalon.

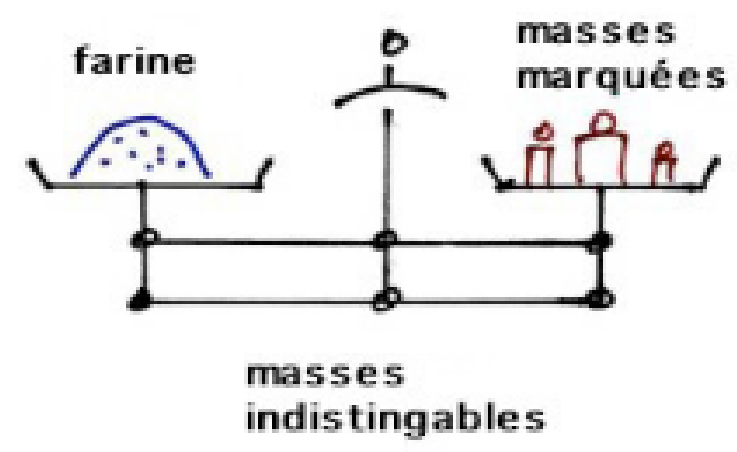

Fig 9 : Balance de Roberval

Autre exemple, la mesure de durée. Le temps n'est pas donné, il est construit par les mouvemements. Un coureur à pied crée un mouvement qui engendre une durée. Si je le chronomètre, mon chronomètre est un autre mouvement, qui construit une autre durée. Je compare ces deux durées de façon indistingable par une coïncidence au départ et une coïncidence à l'arrivée. Il faut avoir une notion de simultanéité qui est une indistingabilité temporelle locale (Einstein, 1905).

Prenons un exemple un peu plus complexe, celui de la balance romaine. Ici, on fabrique une longueur, on fait une comparaison indistingable de couple, on en déduit la masse. Pour graver les traits, on va étalonner, c'est-à-dire qu'on va chercher l'équilibre avec des masses de références sur le plateau et on va graver un trait pour chaque masse de référence. On aura donc là aussi comparé les masses à mesurer à une masse étalon. Mais on 
peut aller plus vite si on connaît la loi des leviers, qui est démontrable à partir d'une hypothèse de symétrie de l'espace (Mach, 1904). Dans ce cas, la fabrication-comparaison aux étalons concerne d'une part la masse fixe (d'un côté du levier) qui est comparée à l'étalon de masse et d'autre part les longueurs (de l'autre côté du levier) qui sont comparées à l'étalon de longueur.

Généralisons. Nous dirons que dans toute mesure, il y a toujours :

1. Fabrication d'un autre objet, dont la connaissance résulte de sa méthode de fabrication,

2. Comparaison indistingable d'une grandeur.

La comparaison peut être indirecte, c'est-à-dire concerner une grandeur différente de celle mesurée, comme dans le cas de la balance romaine. Elle peut s'appuyer sur des lois physiques connues, comme nous l'avons mentionné pour la loi des leviers. (Par exemple, l'algorithme du GPS intègre les équations de la relativité.)

Il n'y a pas de mesure absolue. Il y a comparaison avec des étalons, plus ou moins directement. Les étalons sont fabriqués par les hommes, selon des conventions, et en relation avec les lois physiques considérées comme bien connues.

\section{6. La vision est une mesure}

Étendons notre conception de la mesure à la vision. Voir, c'est construire un autre objet, l'image, qui dans certaines conditions est indistingable de l'objet $v u$. On peut dire aussi : voir, c'est imaginer le monde extérieur (si on met en évidence le radical commun entre image et imaginer). L'image n'est pas forcément matérielle.

Il reste à préciser la nature de cette indistingabilité. Dans le cas de la gravure de Dürer, il s'agit de la superposition-confusion de l'objet et de l'image. Cette superposition est celle permise par la perspective. Nous pouvons donc dire que notre façon de voir est tributaire de cette façon de comparer qui est fondée sur la perspective. Mais il peut s'agir aussi de la superposition-confusion de deux images, ou plus généralement de la comparaison sans superposition entre deux représentations, ou entre un objet et sa représentation, et cela nous permet d'envisager des comparaisons d'indistingabilité qui ne seraient pas reliées à la perspective, car on n'est pas forcé d'utiliser une superposition-confusion et les représentations ne sont pas forcément des images matérielles planes extérieures.

Pour reprendre l'analogie entre vision et mesure, la fabrication de l'image serait la fabrication de l'objet autre, et l'oeil serait l'instrument de comparaison indistingable. Dans ce schéma, la vision serait donc plutôt un acte moteur (de la main ou plus généralement de ses prolongements techniques), et non un acte de l'oeil seul. Le corps du sujet agirait pour transformer en gestes les comparaisons de l'oeil, pour ajuster l'image par itération de la fonction du corps. Si l'on n'agit pas sur le monde extérieur, on ne peut pas voir, de même qu'on ne peut pas mesurer. On peut évoquer ici les mouvements de l'oeil sans lesquels on ne peut pas voir (Yarbus, 1967), en considérant cette action musculaire comme une action sur le monde extérieur.

Ainsi, on comprend également que l'acte de l'artiste nous aide à voir. On ne manque pas de témoignages de peintres ou de photographes qui nous disent qu'ils font ça parce que ça leur permet de voir. Ça leur permet aussi de partager leur vision, ils nous montrent ce qu'ils voient, que nous ne voyons pas sans leur intervention (création). On peut citer Paul Klee : « L'art ne reproduit pas ce qui est visible, mais rend visible » (Klee, 1920).

Dans la perspective, il s'agit de faire que la superposition de l'objet et de l'image devant notre oeil soit comme l'objet seul. C'est la production d'une image qui disparaît lorsqu'elle est superposée à l'objet (à moins que ce ne soit l'objet qui disparaisse). C'est bien ce dont il s'agit dans la gravure de Dürer où ce tableau est un cadre vide. Ce serait une façon particulière de voir, celle utilisée en occident depuis la Renaissance. Elle aurait rendu possible l'apparition de la photographie, comme produisant des représentations matérielles compatibles avec cette façon de voir. Mais il ne fait pas de doute que d'autres civilisations ont eu d'autres façons de voir. Pour s'en convaincre, il suffit d'être attentif aux représentations qu'elles ont créées. Si nous les trouvons parfois moins réalistes, c'est que nous les jugeons avec nos critères de réalisme qui sont liés à la camera obscura. Elles ne sont pas pour autant plus primitives. (Cette remarque commente le verbe conditionner de notre titre qui est mis au sens de Kant.)

\section{7. Comment connaissons-nous l'espace et les objets ?}

Nous devons donc imaginer l'espace et les objets, en testant la cohérence logique de ce que nous avons imaginé. Nous devons aussi mettre ce que nous avons imaginé à l'épreuve des sensations. Car le réel existe, nous ne pouvons pas imaginer l'espace n'importe comment, le réel réagit, on se cogne. Les sensations doivent être en accord avec ce que nous avons imaginé. L'ensemble doit sauver les apparences.

Si nous faisons l'hypothèse la lumière se propage en ligne droite, alors nous pouvons utiliser la fonction 
inverse de la perspective pour imaginer la troisième dimension, la profondeur. Mais la perspective n'est pas inversible. Nous le savons par expérience, car nous savons qu'avec un seul oeil immobile il est difficile de voir la profondeur (nous savons par exemple qu'il est difficile d'enfiler une aiguille). Il nous faut donc nous déplacer dans cet espace des objets (ou bien avoir deux yeux), pour pouvoir faire des recoupements entre plusieurs points de vue.

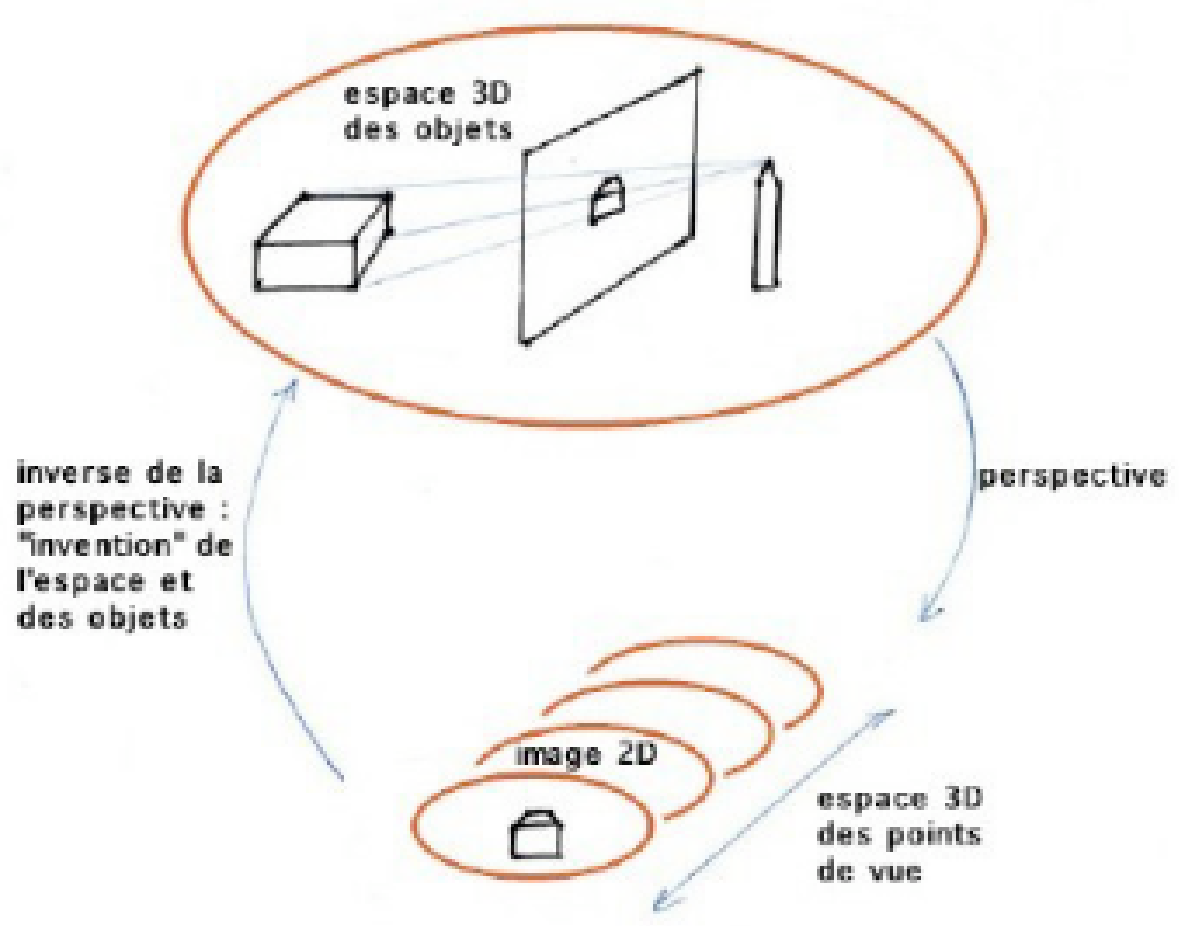

Fig 10 : Schéma de la vision

Nous pouvons ainsi imaginer un espace d'objets à trois dimensions qui soit cohérent. Le travail est le suivant (figure 10) :

1. Imaginer un espace euclidien à 3 dimensions (3D), imaginer que les objets sont situés dans cet espace et les points de vue aussi. À chaque point de vue est associée une image à deux dimensions (2D), en supposant par exemple que cette image est plaquée sur une sphère.

2. Considérer la perspective inverse pour passer des images 2D aux objets en 3 dimensions (invention des objets)

3. Contrôler, par la perspective directe (propagation de la lumière et comparaison par l'oeil), que les objets imaginés redonnent bien les images initiales. Sinon, reboucler la boucle jusqu'à ce que ça colle...

Nous transcrivons ici directement une proposition de Bertrand Russell (Russell, 1914). Russell ne parle pas d'images, mais d'aspects, et pour lui un objet est la classe d'équivalence de ses aspects, de même qu'en arithmétique un nombre entier positif est identifié à la classe d'équivalence des ensembles de même cardinal.

Dans cette conception de Russell, une chose nous semble insatisfaisante : il y a le risque de confondre ces aspects avec des images dont on ne voit pas bien la nature. S'agit-il d'images mentales (qui n'existeraient que dans l'esprit du sujet) ou d'images réelles (avec quelle signification? matérielles ou non ?), dans le monde extérieur ou non (dans le monde des idées de Platon ?), préalables à la vision ou non ? éternelles ou non ? La lecture de son ouvrage Analysis of Mind (Russell, 1921) confirmerait qu'il s'agit d'images intérieures. Il nous semble qu'on peut éviter toutes ces difficultés en rejetant cette notion d'aspect ou d'image et en considérant uniquement notre notion de comparaison d'indistingabilité pour les sensations. Il n'est pas nécessaire de dire la nature de ce qui est indistingable. Ce ne sont pas forcément des images $2 \mathrm{D}$, cela peut être uniquement des propriétés locales aux sensations visuelles. Il suffit de savoir si on distingue ou non, si on perçoit ou non une 
différence. Différence avec quoi ? Avec quelquechose qui est supporté par le langage.

En revanche, la notion de classe d'équivalence nous paraît être la bonne notion pour définir ce qu'est un objet, et dans le sens suivant: si des perceptions multiples (perçues par une même personne ou plusieurs) peuvent être regroupées sous un même discours et porter le même nom, alors il s'agit là de l'apparition d'un objet. La perspective est la métaphore principale pour définir l'objectivité en physique, même dans les théories où les mesures sont très dépendantes de l'observateur (Russell, 1926).

Le sujet imagine l'espace 3D dans lequel se trouvent à la fois l'objet, le point de vue, et éventuellement la représentation (si elle est spatiale). Il doit tester la cohérence de l'ensemble qui inclut sensations, imagination et connaissances verbalisées. La réalité s'introduit ici par le fait que cette cohérence implique les sensations dans la boucle de la figure 10 .

Depuis la relativité d'Einstein, nous savons que la notion d'objets dans l'espace 3D ne tient plus, de même que tout ce qui est dérivé de la notion de substance d'Aristote (Russell, 1925). Néanmoins, notre raisonnement continue à être valide si nous l'adaptons à une notion de perspective dans l'espace-temps relativiste à 4D, mais les entités doivent être adaptées (Russell, 1927).

\section{8. Synthèse d'image et simulation}

Dans la synthèse d'image par ordinateur, les objets et l'espace existent matériellement sous forme d'un codage de nombres dans la mémoire de l'ordinateur. On appelle ça la base de données $3 D$. La perspective est calculée, à l'aide de formules mathématiques décrivant la propagation de la lumière en ligne droite, afin de fabriquer l'image $2 D$. On dit alors que le calcul est une simulation, et qu'on fait une synthèse d'image, par analogie probablement avec la synthèse chimique, dans laquelle on construit des molécules suivant le modèle de ce qui existe déjà dans la nature, mais en utilisant d'autres voies, artificielles.

On pourrait faire un schéma de la synthèse d'images. Il serait identique à celui de la figure 10, dans lequel la base de données 3D se substituerait à l'espace des objets et le calcul de la perspective se substituerait à la propagation de la lumière. Le schéma serait exactement le même que pour la vision. L'oeil intervient pour tester si l'image lui convient, soit parce qu'elle évoque du déjà $v u$, soit parce qu'au contraire elle permet d'aller vers la fantaisie.

Dans le contexte informatique, c'est le caractère (soit-disant) immatériel de la base de données ainsi que le calcul (simulation) qui font qualifier de virtuels ces objets. Mais est-ce différent de la vision précédemment décrite ? En quoi l'espace des objets de la réalité est-il plus réel ? ou plus matériel ? en quoi la perspective faite par la lumière (dont la propagation est imaginée) est-elle plus tangible que celle faite par le calcul ?

Certes, la simulation nous permet d'imaginer des mondes non réalistes, c'est-à-dire des situations que nous n'avons jamais rencontrées dans le monde habituel et que l'on peut qualifier de fantaisies. Mais pouvons nous parier qu'il s'agit de situations que nous ne rencontrerons jamais ? Les seuls cas où nous pouvons en être sûrs, c'est lorsqu'il y a une contradiction flagrante entre un monde imaginé et l'expérience.

Nous arrivons ainsi à une définition $d u$ réel. Nous pouvons dire que le réel est de l'imaginaire qui est indistingable de l'expérience. Avec cette définition, le réel serait un cas particulier du virtuel : le réel, il faut l'inventer et le simuler, et à ce titre, c'est du virtuel. Mais il réagit, il ne se laisse pas imaginer n'importe comment. Ce serait donc un sous-ensemble particulier de ce qui est imaginable.

Mais on peut aussi dire les choses autrement, et déboucher sur une autre définition du réel. On peut rejeter le réel en dehors de ce qui est synthétisé. Dans tous les cas où ce qui est synthétisé ou imaginé est conforme à l'expérience, alors on n'appellera pas cela réel, mais imaginaire. Par contre, si on n'arrive pas à inventer un modèle qui permette de produire quelquechose conforme à l'observation, alors ce qui est observé sera appelé réel. Avec cette définition, le réel serait au-delà du calculé, au-delà du connu.

\section{9. La physique et la mesure}

Le physicien élabore des théories et des modèles (conceptuels) et en calcule les conséquences (simulation). Si ces conséquences sont indistingables de la réalité, alors il dit que ses théories sont satisfaisantes. Comment peut-il vérifier? On nous dit que c'est en faisant des mesures et en comparant les résultats de mesure aux résultats de simulation. Mais une mesure est déjà construction d'un objet et comparaison. En fait, la simulationcomparaison est déjà dans la mesure ! Pour mieux l'appréhender, faisons le schéma de la mesure, figure 11.

Les trois schémas, de la vision, de la simulation, et de la mesure sont identiques. Dans tous les cas, l'espace $3 D$ et les objets sont imaginés.

Prenons l'exemple qui est à l'origine des sciences de la nature, l'image du ciel étoilé. À partir des lois de la 
physique et des paramètres des mouvements de tous les corps célestes, on calcule (simulation ou synthèse) l'image du ciel à un instant spécifié. Si il y a une différence entre cette image et l'observation, alors on modifie le programme de l'ordinateur ou les paramètres des mouvements. Dans le cas où on arrive à ce qu'il n'y ait plus de différence distingable entre les images du ciel (vues par l'intermédiaire des instruments de mesure) et les images du ciel calculées par la simulation, alors en quoi le ciel de la réalité est-il différent du ciel simulé ? (Il n'y a alors plus de réel, si on a défini le réel comme ce qui n'est pas simulé.)

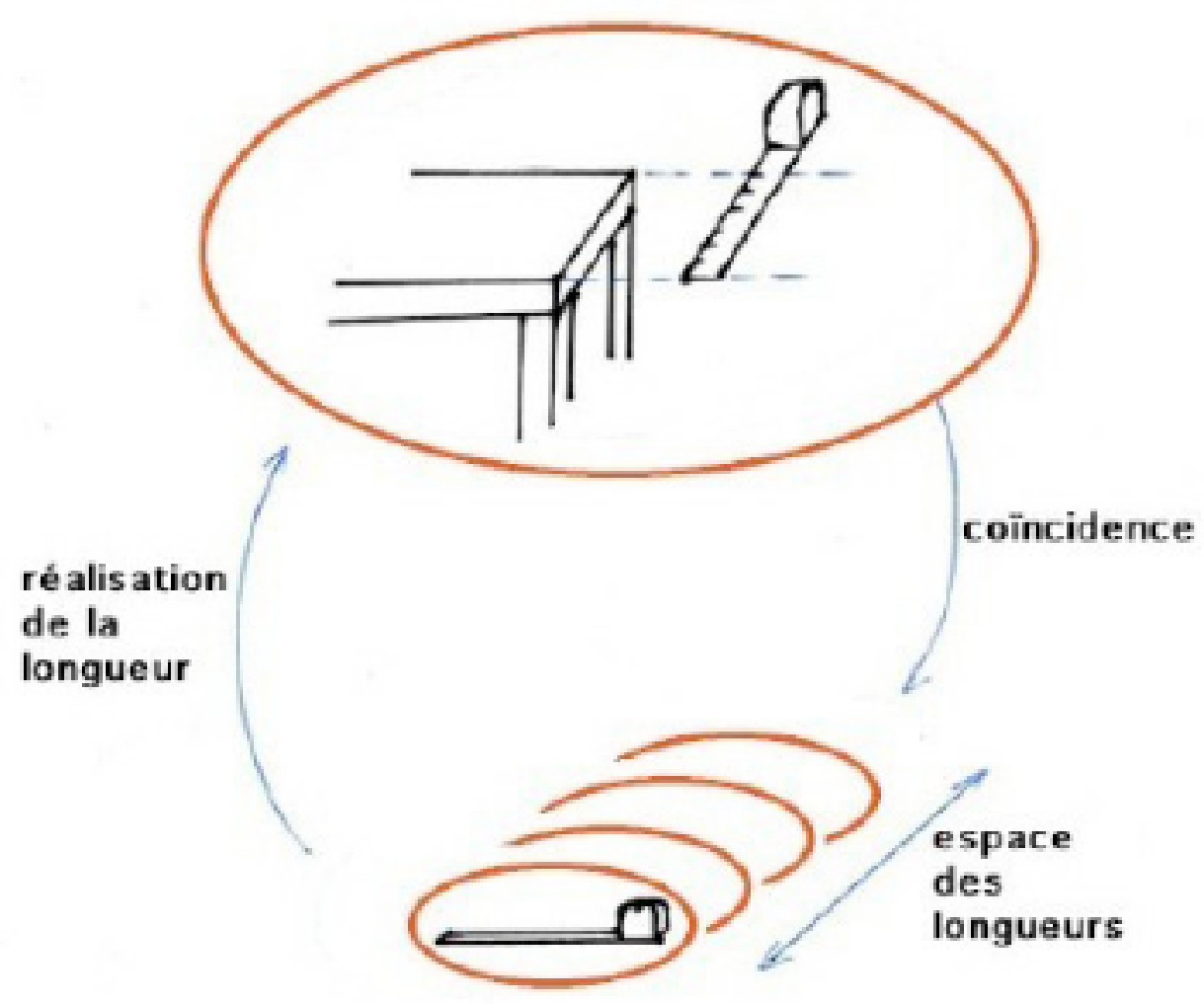

Fig 11 : Schéma de la mesure en physique

En quoi l'informatique et le numérique seraient davantage virtuels? Il y a toujours eu de l'imagination et du calcul dans la vision et dans la physique. Il y a toujours eu de la simulation. Ce qui est nouveau est la puissance de calcul des ordinateurs qui nous permet d'accéder à des simulations jusqu'alors impossibles, et qui permet l'interactivité dans le cas de simulations dynamiques (rapides). Cette interactivité change qualitativement notre perception des mondes fantaisistes. Puisqu'elle nous les rend réalistes, nous les qualifions de virtuels pour essayer de dire qu'ils ne sont peut-être pas la réalité. On ne peut en décider que lorsqu'on se cogne sur de l'imprévu, sur du réel.

\section{CONCLUSION}

Nous avons été conduit à nous interroger sur l'essence des objets et de l'espace, et nous avons montré comment ces objets sont le résultat d'une construction, d'une extrapolation, sont produits par l'imagination. Cela n'enlève rien à leur réalité puisque cette construction doit être compatible avec les sensations.

Nous avons raisonné en utilisant l'entité lumière et nous lui avons attribué un double statut :

1. D'une part, la lumière se propage dans l'espace-temps. L'espace-temps apparaît alors comme un préalable, un contenant pour la lumière. 
2. D'autre part, l'hypothèse faite sur la propagation de la lumière permet d'imaginer, de construire l'espace, le temps et les objets. La lumière apparaît ainsi comme un préalable à la construction de l'espace-temps.

La lumière semble prise dans une apparente contradiction. Chacune des deux parties de notre texte illustre une des deux positions qui peuvent apparaître comme contradictoires. Mais cette contradiction disparaît si nous admettons que ces deux attitudes sont enchaînées dans une boucle algorithmique telle que celle que nous avons présentée pour la construction du monde extérieur. Ce n'est plus une contradiction, c'est une construction itérative.

Nous avons choisi un cadre classique pour cette description : d'une part, l'espace à trois dimensions est celui de la physique de Newton, d'autre part la propagation de la lumière est celle de l'optique géométrique. Dans ce cadre, l'espace de Newton est un contenant vide dans lequel la lumière se propage, et voir demande à utiliser la propagation de la lumière en ligne droite pour construire les objets. Dans les théories physiques plus récentes, les ingrédients ne sont plus les mêmes, mais il nous semble que la boucle logique se déplace sans disparaître. Les statuts logiques évoluent, mais l'ensemble du discours ne peut être purement axiomatique, reposant sur des entités premières, ou éléments, jouant le rôle d'axiomes. Le langage est toujours dans la boucle.

La façon qu'a notre société de voir l'espace et les objets serait donc reliée à notre façon de construire des images à l'aide de la perspective, et le lien entre les deux peut être appréhendé en interrogeant le statut logique de propositions de physique comme celles concernant la propagation de la lumière. Il nous semble qu'une autre société, avec un autre langage, qui construit ses images autrement, qui construit sa physique autrement, voit autrement. Ainsi la camera obscura structure la pensée occidentale moderne. La notion même de monde extérieur n'est-elle pas issue de cette séparation effectuée par la cloison de la chambre entre un dedans et un dehors? Ne serait-ce pas cette chambre qui nous pousse à penser nos représentations comme intérieures ? C'est suggéré par Lacan dans son séminaire, à propos de l'idéalisme de Berkeley (Lacan, 1969).

Y aurait-il une façon de voir meilleure que d'autres ? Ce pourrait être une question de physicien qui cherche à améliorer ses théories. Il nous semble que l'existence de la boucle logique que nous avons mentionnée, et qui nous apparaît nécessaire, enlève toute motivation pour une telle question. La science ne peut que chercher à étendre le domaine de cohérence logique de son discours, en simplifiant ses équations pour atteindre une plus grande élégance, mais cet édifice pourra à tout moment être remplacé par un autre, qui pourrait être très différent. Un peu comme en mathématiques une nouvelle démonstration d'un théorème connu peut emprunter un chemin très différent des démonstrations précédentes ; la signification qu'on pourrait vouloir y attacher pourrait en être complètement modifiée.

\section{Remerciements}

Ce texte est issu d'une réflexion menée à l'occasion de l'exposé prononcé le 9 février 2005 au séminaire de photographie de l'école normale supérieure (Matherat, 2005), sous le titre « Tracer le chemin de la physique à la métaphysique - le virtuel est-il où on l'attend ? ». Ce séminaire, dirigé par Monique Sicard, avait cette année-là pour titre «Latent, virtuel». Notre exposé avait été l'occasion de dire notre façon de comprendre l'ouvrage « La fabrique du regard » (Sicard, 1998), en le mettant en relation (à notre manière) avec les conceptions de Bertrand Russell dans « Our knowledge of the external world» (Russell, 1914). Au-delà d'une réflexion sur la vision, ce travail est motivé par notre recherche en électronique numérique, dans lequel nous sommes amenés à nous interroger sur les rapports entre l'être et le langage des composants électroniques élémentaires, par exemple lorsqu'il s'agit de relier une propriété physique d'un composant à sa fonction logique (Matherat et Jaekel, 1996).

Nous remercions Claude Lothier, auteur de l'exposition « Persistante Perspective» (Lothier, 2004), pour son intervention dans le séminaire de photographie et nos fertiles discussions à propos de la perspective.

Nous remercions les lecteurs de la revue qui nous ont aidé à améliorer le texte en pointant les ambigüités de terminologie si piégeantes dans ce domaine.

\section{Références bibliographiques}

Einstein A. (1905). Zur Elektrodynamik bewegter Körper, Annalen der Physik, Vol. 17, p. 891-921.

Feynman R. (1963). Mécanique 2, Le cours de physique de Feynman, Mécanique deuxième partie, traduction française, Interéditions 1979.

Frizot M. (1994). Nouvelle histoire de la photographie, ouvrage collectif sous la direction de Michel Frizot, Bordas.

Gaudart L. et Albet M. (1997). Physique photographique, Le temps apprivoisé.

Kant E. (1783). Prolégomènes à toute métaphysique future qui pourra se présenter comme science, 
traduction de Louis Guillermit, Librairie philosophique Vrin, 1986, poche 1993.

Klee P. (1920). Confession d'un créateur, extrait de l'ouvrage édité par Taschen et le journal Le Monde sur Paul Klee en 2006.

Lacan J. (séance du 30 avril 1969). Dedans dehors, in D'un Autre à l'autre, Le séminaire livre 16, éditions du Seuil, mars 2006.

Lothier C. (2004). Exposition Persistante Perspective, présentée en 2004 à l'École supérieure des beaux-arts du Mans.

Mach E. (1904). La Mécanique, Hermann, réédition Jacques Gabay 1987.

Matherat P. et Jaekel M.-T. (1996). Dissipation logique des implémentations d'automates - dissipation du calcul, Technique et Science Informatiques, 15, 8, 1079-1104, et "http://perso.enst.fr/ matherat/publications/tsi96/".

Matherat P. (2005). Site web du séminaire de photographie de l'école normale supérieure, "http://seminaire.photo.ens.free.fr/".

Matherat P. (2006). Qu'est-ce que voir? Ou comment notre vision est conditionnée par la camera obscura, "http://halshs.archives-ouvertes.fr/halshs-00109018".

Platon, La république, livre VII.

Russell B. (1914). Our knowledge of the external world, réédition Routledge 1993.

Russell B. (1921). The Analysis of Mind, réédition Routledge 1996.

Russell B. (1925). ABC of relativity, réédition Routledge 1997.

Russell B. (1926). Philosophical Consequences of Relativity, Britannica, 13ème édition. Accessible à "http://www.britannica.com/heritage/article?content_id=1368".

Russell B. (1927). The Analysis of Matter, réédition Routledge 1992.

Sicard M. (1998). La fabrique du regard, Odile Jacob.

Yarbus A. L. (1967). Eye movements and vision, New York : Plenum press. 